\title{
Contrast Mechanism for Visualization of Ferroelectric Domains with Scanning Force Microscopy
}

\author{
T. JUNGK* AND E. SOERGEL \\ Institute of Physics, University of Bonn, Wegelerstraße 8, \\ D-53115 Bonn, Germany
}

\begin{abstract}
Scanning force microscopy is an ideal technique for non-destructive investigation offerroelectric domains. Application of such domains for efficient optical frequency conversion and for ultrahigh-density data storage requires $\mu \mathrm{m}$ to $\mathrm{nm}$-sized domain structures. Thus the high lateral resolution offered by scanning force microscopy is highly desired. However, the mechanism causing the domain contrast is not yet fully understood. Investigations of periodically-poled lithium niobate samples with conductive as well as insulating surface layers indicate that the observed domain contrast is not solely due to the electromechanical piezoresponse.
\end{abstract}

Keywords Scanning force microscopy; domains visualization; $\mathrm{LiNbO}_{3}$

\section{Introduction}

Domain engineering in lithium niobate $\left(\mathrm{LiNbO}_{3}\right)$ crystals has become important for many application areas such as photonics or high-density data storage. Periodically-poled crystals (PPLN) facilitate nonlinear-optical applications for second harmonic generation and optical parametrical oscillation through quasi phase matching [1-3], and tailored domain structures enable electro-optically tunable diffractive-optical elements [4]. The use of ferroelectric domain patterns as nonlinear photonic crystals is another very promising prospect [5], and first results were obtained for a combined photorefractive/nonlinear-optical device namely a PPLN optical parametrical oscillator with a photorefractive grating as a distributed feedback Bragg reflector [6]. Furthermore high-density data storage was realized by writing and reading of nanodomains in ultra-thin $(\sim 100 \mathrm{~nm})$ crystals [7].

For optimization of the particular domain structures there is need for a simple visualization method with high spatial resolution [8]. Several techniques have been used such as toning [9], near-field optical microscopy [10], or scanning electron microscopy [11], but the most widely utilized method is selective chemical etching [12] because of its comparative ease of use. The obvious disadvantage of the latter is that it is a destructive technique. More recently, scanning force microscopy (SFM) has been established as an optimum tool for the nondestructive visualization of ferroelectric domains with high lateral resolution [13]. However, the contrast mechanism is not yet fully understood. In this contribution, we

Paper originally presented at IMF-11, Iguassu Falls, Brazil, September 5-9, 2005; received for publication January 26, 2006.

${ }^{*}$ Corresponding author. E-mail: jungt@ @ physik.uui-bonn.de 
report on investigations of PPLN crystals covered with conducting as well as insulating layers.

\section{Experimental}

The experiments were carried out with congruently melting z-cut $\mathrm{LiNbO}_{3}$ crystals (thickness $500 \mu \mathrm{m}$ ) with periodically-poled structures (period length 8-30 $\mu \mathrm{m}$ ). In our experiments we used a commercial SFM (SMENA, NT-MDT) that has been modified to allow application of voltages to the tip. We used gold-coated tips and operated the instrument as a dynamic contact electrostatic force microscope (DC-EFM) [14], i.e. the measurements were performed in contact mode with an additional $\mathrm{AC}$ voltage $\left(10 \mathrm{~V}_{\mathrm{pp}}, 36 \mathrm{kHz}\right)$ applied to the tip. The time constant of the feedback circuit is very large compared to the period of modulation of the applied voltage, and thus the topography can be read out as in standard contact-mode SFM. To get information about the domain structure of the samples, we record in addition to the topography the AC-voltage driven answer of the cantilever via lock-in detection. The phase of the lock-in amplifier is adjusted such that the $+\mathrm{z}(-\mathrm{z})$ faces appear as dark (bright) areas in the images.

\section{Results}

To demonstrate the capabilities of our experimental setup, in Fig. 1 we show a scan of a PPLN structure with an underlying titanium in-diffused waveguide [15]. For the observation of the domain contrast we recorded the oscillation amplitude of the cantilever caused by forces normal to the surface (Fig. 1a). The simultaneously recorded topography (Fig. 1b) shows the in-diffused waveguide running perpendicularly to the domain stripes. Please note that the two signals are almost completely separated, i.e. just a very faint shadow of the topography can be seen in the domain image.

In the literature the contrast observed in DC-EFM images like that in Fig. 1a is usually explained via the piezoelectric deformation of the sample, i.e. the crystal is expanding or contracting depending on the orientation of the polar axis and the polarity of the applied voltage. The expected thickness change $\Delta z$ can be calculated according to $\Delta z=\left(e_{33} / C_{33}\right)$ $U$. Here $e_{33}=1.785 \mathrm{C} / \mathrm{m}^{2}$ denotes the corresponding longitudinal piezoelectric tensor element [16], $C_{33}=2.357 \times 10^{11} \mathrm{~N} / \mathrm{mm}^{2}$ the appropriate stiffness tensor element [16], and $U$ the voltage applied to the crystal between tip and the back-side electrode. Applying $U=10 \mathrm{~V}_{\mathrm{pp}}$ results in a thickness change of $76 \mathrm{pm}$. The measured values, however, are larger up to one order of magnitude and highly depend on the chosen $\mathrm{AC}$ frequency.

For comparison we measured the piezoelectric thickness changes of the crystal by applying high DC voltages $( \pm 200 \mathrm{~V})$ to the tip. Fig. 2 shows the piezoelectric deformation
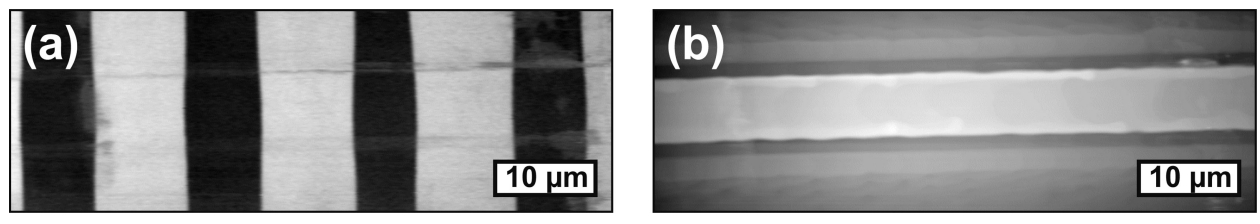

Figure 1. Simultaneously recorded DC-EFM images of the domain structure (a) and the topography (b) of PPLN with a waveguide underneath. The two signals are almost completely separated. The sample is by courtesy of Prof. W. Sohler, Paderborn. 


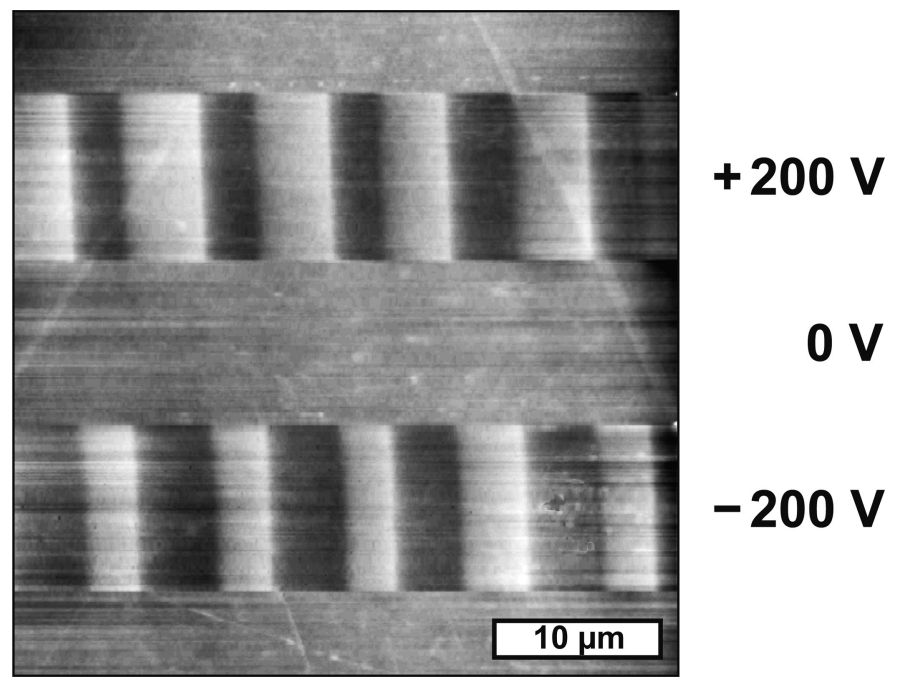

Figure 2. Topography of a PPLN crystal with areas where voltages of $\pm 200 \mathrm{~V}$ were applied between tip and back electrode during the scanning.

of the sample. Changing the polarity of the applied voltage inverts the observed domain contrast. The height of the surface steps of $1.5 \pm 0.1 \mathrm{~nm}$ is in very good agreement with the calculated value of $1.5 \mathrm{~nm}$ for $200 \mathrm{~V}$. Note that the inhomogeneous field distribution at the tip has no influence on the resulting piezoelectric deformation of the sample [17].

For further understanding of the detection mechanism we covered PPLN samples with conducting as well as dielectric layers. The conducting layers consist of very thin films of copper $(5-55 \mathrm{~nm})$ that were deposited by physical vapor deposition on the samples through different masks with openings between 1-2000 $\mu \mathrm{m}^{2}$. It turned out that for copper the domain contrast in DC-EFM decreases drastically already for layers of few nm thickness and vanishes completely as the films become conductive $(>10 \mathrm{~nm})$. The signal also breaks down if only an island on top of a single domain is metallized; no short-circuit of two adjacent domains is required (Fig. 3).

When covering PPLN samples with dielectric $\mathrm{SiO}_{2}$ films we see a quite different behavior: The domain contrast decreases substantially only at film thicknesses of more than $50 \mathrm{~nm}$ and is still visible through layers of nearly $1 \mu \mathrm{m}$ (Fig. 4). The decrease of the domain contrast follows an inverse square root behavior of the dielectric layer thickness as can be seen from the graph in Fig. 4. Although the contrast diminishes with increasing film thickness, the lateral resolution of the detection mechanism, i.e. the slope at the domain boundaries, is only slightly affected.

\section{Discussion}

The high DC voltage experiments demonstrate very clearly that for this mode of operation the mechanism for domain visualization relies on the converse piezoelectric effect. For the DC-EFM measurements with moderate AC voltages this is not quite clear as the measured oscillation amplitude of the cantilever does not correspond to the calculated value and above all depends on the chosen frequency. With conductive copper layers on top of the PPLN crystals the domain contrast breaks down and the oscillation amplitude corresponds 


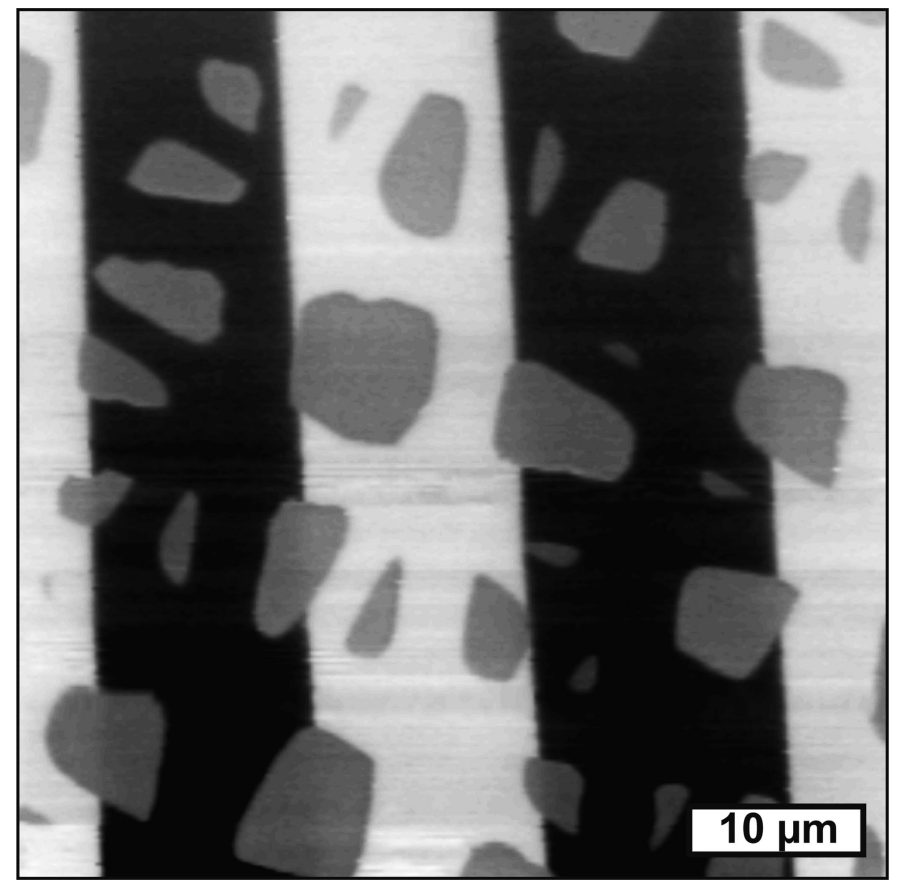

Figure 3. DC-EFM image of PPLN with copper islands of $40 \mathrm{~nm}$ thickness on which the domain contrast is suppressed.

to the average of the amplitudes on the $\pm c$ domains. This result is independent of neither the lateral size nor position of the coated areas, which is contradictory to a piezoelectric response, as the deformation of the sample must not depend on the electrode shape nor size. On the other hand the insulating, dielectric coating does not suppress the piezoelectric response of the crystal but attenuates the domain contrast with the square root of the layer thickness according to the visible depth for SFM. From both results we conclude that
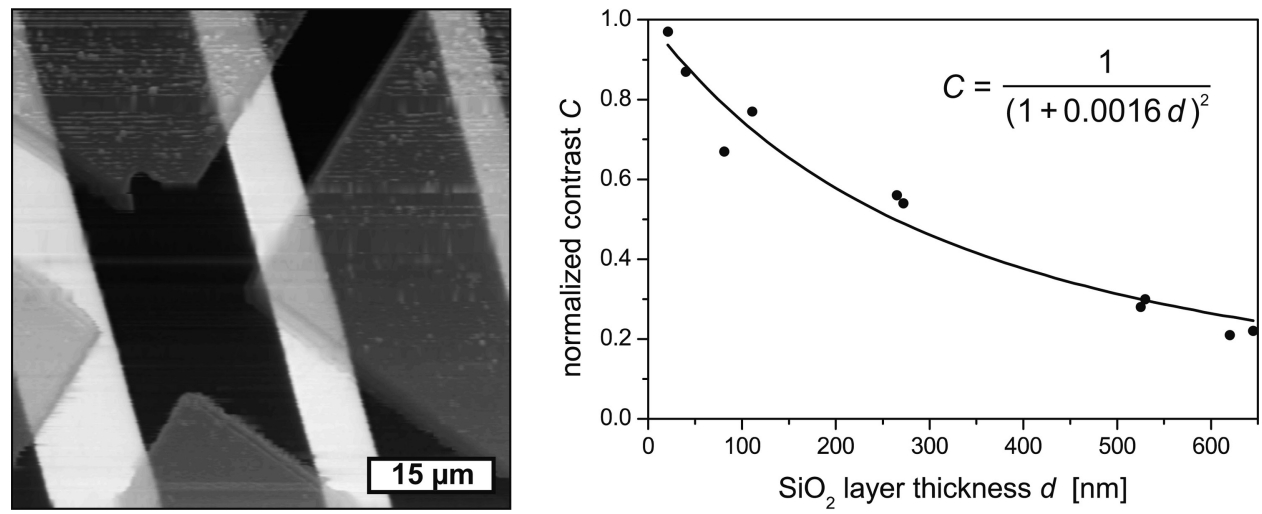

Figure 4. DC-EFM image of PPLN with $\mathrm{SiO}_{2}$ coated areas of $85 \mathrm{~nm}$ thickness. If the normalized contrast is plotted against the $\mathrm{SiO}_{2}$ layer thickness one gets an inverse square root behavior of the contrast. 
the DC-EFM signal cannot solely depend on the converse piezoelectric effect but also on some electrostatic interactions as the signal from the surface layers disagrees with static measurements of contacted and insulated piezoelectric crystals. The signals are presumably also strongly inflated by resonances of the scanning system, which will be analyzed in detail soon.

\section{Conclusion}

In this paper we have presented investigations regarding the contrast mechanism for the detection of ferroelectric domains with SFM. Direct measurements of the piezoelectric effect in $\mathrm{LiNbO}_{3}$ have been shown by high-voltage SFM. In addition, the influence of metallic and dielectric layers on the domain visualization in DC-EFM is analyzed. It indicates that the domain contrast is not solely due to the converse piezoelectric effect and mechanical resonances probably enhance the domain contrast. Although the contrast mechanism is not yet fully understood, these experiments will help to achieve a thorough explanation.

\section{Acknowledgment}

Financial support of the DFG research unit 557 and of the Deutsche Telekom AG is gratefully acknowledged.

\section{References}

1. M. Yamada, N. Nada, M. Saitoh, and K. Watanabe, First-order quasi-phase matched $\mathrm{LiNbO}_{3}$ waveguide periodically poled by applying an external field for efficient blue second-harmonic generation. Appl. Phys. Lett. 62, 435-436 (1993).

2. E. Myers, G. D. Miller, R. C. Eckardt, M. M. Fejer, R. L. Byer, and W. R. Bosenberg, Quasiphase-matched optical parametric oscillators in bulk periodically poled $\mathrm{LiNbO}_{3}$. Opt. Lett. $\mathbf{2 0}$, 52-54 (1995).

3. M. M. Fejer, G. A. Magel, D. H. Jundt, and R. L. Byer, Quasi-phase-matched second harmonic generation: tuning and tolerances. IEEE J. Quantum Elect. 28, 2631-2654 (1992).

4. J. A. Abernethy, C. B. E. Gawith, R. W. Eason, and P. G. R. Smith, Demonstration and optical characteristics of electro-optic Bragg modulators in periodically poled lithium niobate in the near-infrared. Appl. Phys. Lett. 81, 2514-2516 (2002).

5. N. G. R. Broderick, G. W. Ross, H. L. Offerhaus, D. J. Richardson, and D. C. Hanna, Hexagonally poled lithium niobate: a two-dimensional nonlinear photonic crystal. Phys. Rev. Lett. 84, 43454348 (2000).

6. A. C. Chiang, Y. Y. Lin, T. D. Wang, and Y. C. Huang, Distributed-feedback optical parametric oscillation by use of a photorefractive grating in periodically poled lithium niobate. Opt. Lett. 27, 1815-1817 (2002).

7. Y. Cho, K. Fujimoto, Y. Hiranaga, Y. Wagatsuma, A. Onoe, K. Terabe, and K. Kitamura, Tbit/inch ${ }^{2}$ ferroelectric data storage based on scanning nonlinear dielectric microscopy. Appl. Phys. Lett. 81, 4401-4403 (2002).

8. E. Soergel, Visualization of ferroelectric domains in bulk single crystals. Appl. Phys. B 81, 729-752 (2005).

9. M. Houé and P. D. Townsend, An introduction to methods of periodic poling for second-harmonic generation. J. Phys. D 28, 1747-1763 (1995).

10. T. J. Yang, V. Gopalan, P. J. Swart, and U. Mohideen, Direct observation of pinning and bowing of a single ferroelectric domain wall. Phys. Rev. Lett. 82, 4106-4109 (1999).

11. G. Y. Robinson and R. M. White, Scanning electron microscopy of ferroelectric domains in barium titanate. Appl. Phys. Lett. 10, 320-323 (1967). 
12. C. L. Sones, S. Mailis, W. S. Brocklesby, R. W. Eason, and J. R. Owen, Differential etch rates in z-cut $\mathrm{LiNbO}_{3}$ for variable $\mathrm{HF} / \mathrm{HNO}_{3}$ concentrations. J. Mater. Chem. 12, 295-298 (2002).

13. M. Alexe and A. Gruverman, Nanoscale characterisation of ferroelectric materials. Berlin, New York: Springer, 1st edition (2004).

14. J. W. Hong, K. H. Noh, S. Park, S. I. Kwun, and Z. G. Khim, Surface charge density and evolution of domain structure in triglycine sulfate determined by electrostatic-force microscopy. Phys. Rev. $B$ 58, 5078-5084 (1998).

15. G. Schreiber, H. Suche, Y. L. Lee, W. Grundkötter, V. Quiring, R. Ricken, and W. Sohler, Efficient cascaded difference frequency conversion in periodically poled $\mathrm{Ti}: \mathrm{LiNbO}_{3}$ waveguides using pulsed and cw pumping. Appl. Phys. B 73, 501-504 (2001).

16. M. Jazbinšek and M. Zgonic, Material tensor parameters of $\mathrm{LiNbO}_{3}$ relevant for electro- and elasto-optics. Appl. Phys. B 74, 407-414 (2002).

17. H. N. Lin, S. H. Chen, S. T. Ho, P. R. Chen, and I. N. Lin, Comparative measurements of the piezoelectric coefficient of a lead zirconate titanate film by piezoresponse force microscopy using electrically characterized tips. J. Vac. Sci. Technol. B 21, 916-918 (2003). 